For Internal Distribution Only

Accelerator Division

Alternating Gradient Synchrotron Department

BROOKHAVEN NATIONAL LABORATORY

Upton, New York 11973

Accelerator Division

Technical Note

AGS/AD/Tech. Note No. 455

AGS Tune Quad Production Measurements

E. Bleser

January 14,1997 


\title{
AGS TUNE QUAD PRODUCTION MEASUREMENTS
}

\author{
E. BLESER
}

\section{INTRODUCTION}

This note reports on the magnetic measurements made on the high field quadrupoles which were installed in the AGS in the early 1990's as the Horizontal and Vertical Tune Quads and as the Skew Quads. It consists of three parts. Part A summarizes the available measurements. It includes results on 22 of the 30 quads installed. Part B is an example of a detailed report which is generated for each magnet. These reports will not be given wide circulation, but they will be stored as part of the permanent record for each magnet. Part $\mathrm{C}$ is a data sheet for these magnets.

\section{A. SUMMARY OF RESULTS}

This note reports on results from 22 measured quadrupoles. The magnets were measured by the $\mathrm{AD}$ Group and the results were reported in their TMG Series of notes as well as being made available to us on the VAX computer.

The nomenclature we shall use is as follows:

$$
\begin{aligned}
& B_{y}(X)=B_{0}+B_{1} * X+B_{2} * X^{2}+B_{3} * X^{3}+\ldots \\
& B_{x}(X)=A_{0}+A_{1} * X+A_{2} * X^{2}+A_{3} * X^{3}+\ldots .
\end{aligned}
$$

In a quadrupole the only allowed terms are $\mathrm{B}_{1}$ and $\mathrm{B}_{5}$ etc. Those magnets installed as skew quads are rotated 45 degrees in effect interchanging $\mathrm{Al}$ and $\mathrm{B} 1$.

All the measurements are DC, and are made with a rotating coil, 36.5 inches long, which projects well outside the ends of the magnets. Therefore all our data is in the form of integrated field values, written as $\mathrm{B}_{1}{ }^{*} \mathrm{~L}_{\text {eff }}$ etc. Figure 1 shows a typical plot of $\mathrm{B}_{1}{ }^{*} \mathrm{~L}_{\text {eff }}$, the integrated gradient, versus the current, I. Figure 2 is a more interesting plot of the integrated gradient divided by I versus I. The simple linear fit shown in Figure 1 does not give a good fit to the data when it is plotted on the greatly expanded scale of Figure 2. The fit shown in Figure 2 is derived from a sixth power fit to the plot in Figure 1.

$$
B 1 * L_{\text {eff }}=q_{0}+q_{1}^{*} I+q_{2}^{*} I^{2}+q_{3}^{*} I^{3}+q_{4}^{*} I^{4}+q_{5}^{*} I^{5}+q_{6}^{*} I^{6}
$$

Table 1 gives the results of this fit. 


\begin{tabular}{||c|c||}
\hline \multicolumn{2}{|c|}{ TABLE 1 } \\
\hline \hline q0 & $1.7718 \mathrm{E}-03$ \\
\hline q1 & $1.7193 \mathrm{E}-3$ \\
\hline q2 & $6.3848 \mathrm{E}-8$ \\
\hline q3 & $-6.4030 \mathrm{E}-11$ \\
\hline q4 & $-1.1111 \mathrm{E}-13$ \\
\hline q5 & $2.5804 \mathrm{E}-16$ \\
\hline q6 & $-1.3982 \mathrm{E}-19$ \\
\hline
\end{tabular}

The precision used here may seem excessive but something of this sort is needed to give a good fit in Figure 2, the classic form of presenting precision magnet data. In this case there is very little saturation but a very visible residual field effect. The relative measurement accuracy has been reported in previous results (Booster Technical Note 174) as one part in ten thousand. This applies to all of these quads. The absolute measurement accuracy (essentially the area of the measuring coil) must be known to compare these quads against the AGS main magnets but has not been calibrated and at present can be estimated to be accurate to one or two per cent.

Figure 3 is a plot of $\mathrm{B} 1 * \mathrm{Leff} / \mathrm{I}$ at 500 and at 800 Amperes for the 22 measured magnets. It shows that at 500 Amperes the average for the measured magnets is:

$$
B 1^{*} L_{\text {eff }} / \mathrm{I}=0.001735 \pm 0.000003 \mathrm{~T} / \mathrm{A}
$$

and that the saturation in each magnet is very similar from magnet to magnet. Magnet number 18 matches this average value and will be used to typify the entire collection of magnets. The first allowed term, B5, is so small that the results do not seem reportable.

\section{B. STANDARD MEASUREMENT REPORT}

The appended report has been generated and permanently stored for each magnet. It is intended to be self-explanatory. 


\section{PARAMETER SHEET FOR THE AGS TUNE QUAD}

The appended data sheet is an attempt to provide a fairly complete description of a magnet.

\section{ACKNOWLEDGMENTS}

The analysis and the conclusions in this note are the responsibility of the author alone and represent his sole contribution to this effort. The measurements were carried out by the Measurements Group of the Accelerator Development Division, using a system developed over many years by many people, with a particular effort having been expended over the past several years to adapt the system to the present application. 


\section{B1*Leff vs I}

QNU018

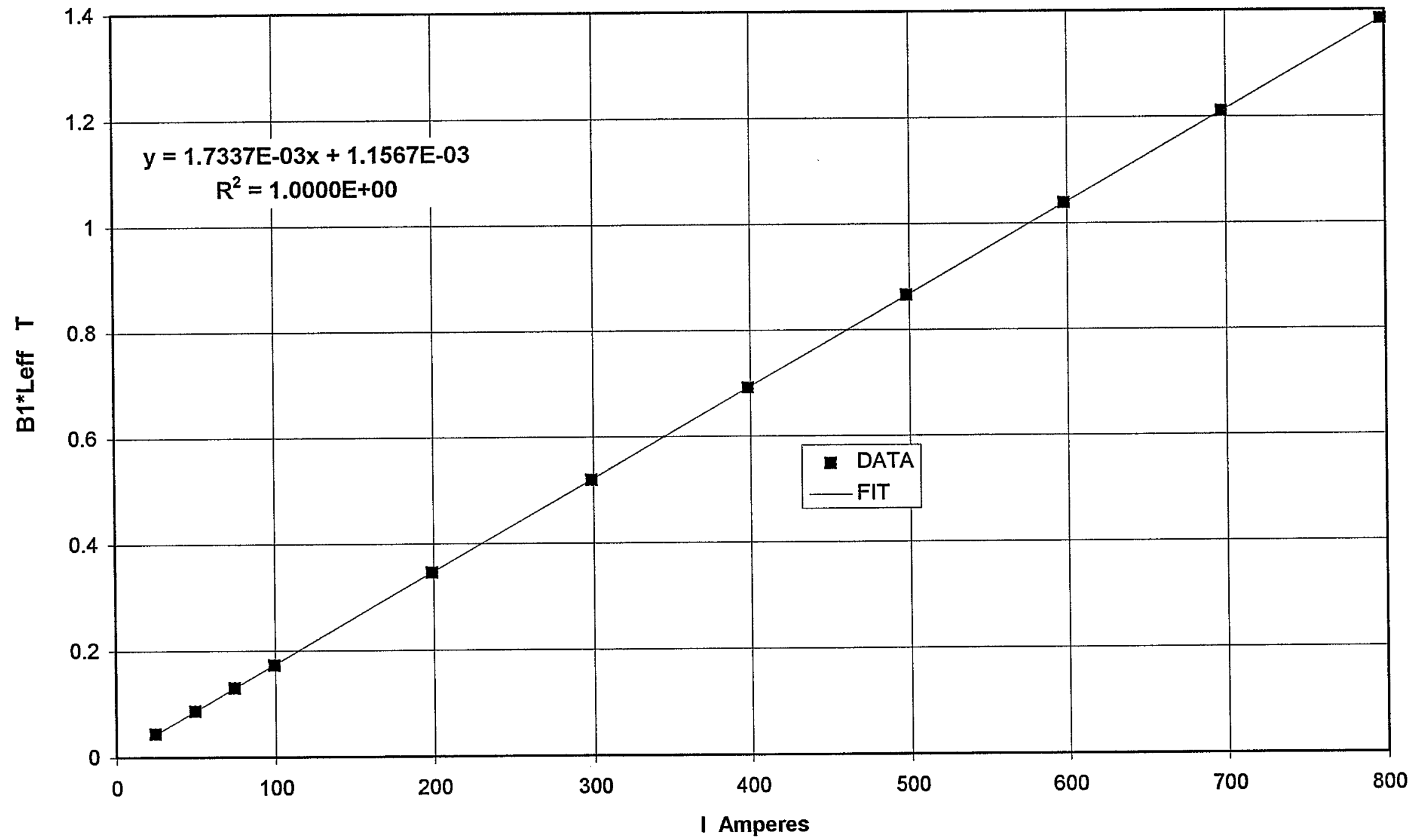


Figure 2

\section{B1*Leff / I vs I}

QNU018

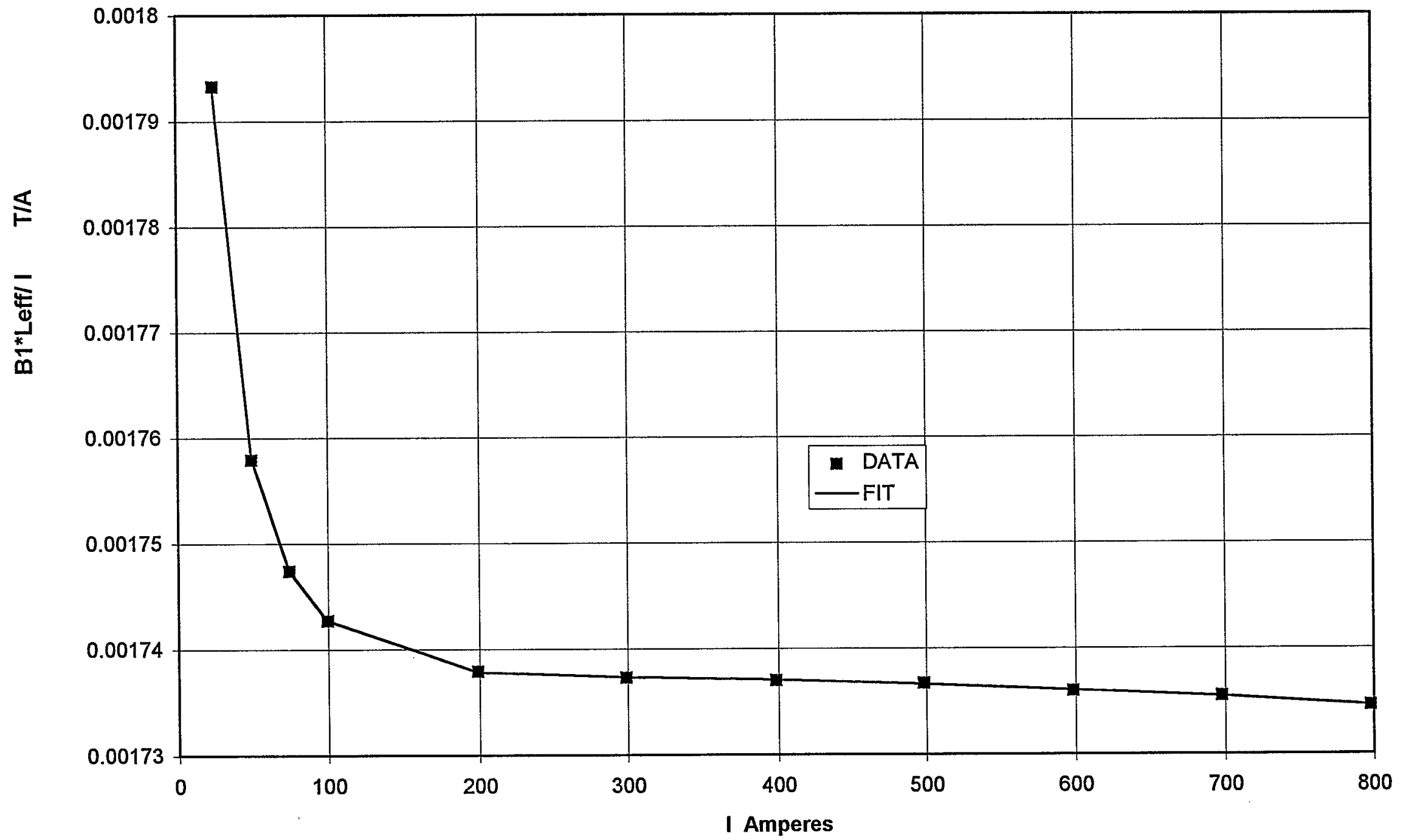




\section{B1*Leff / / vS MAGNET NUMBER}

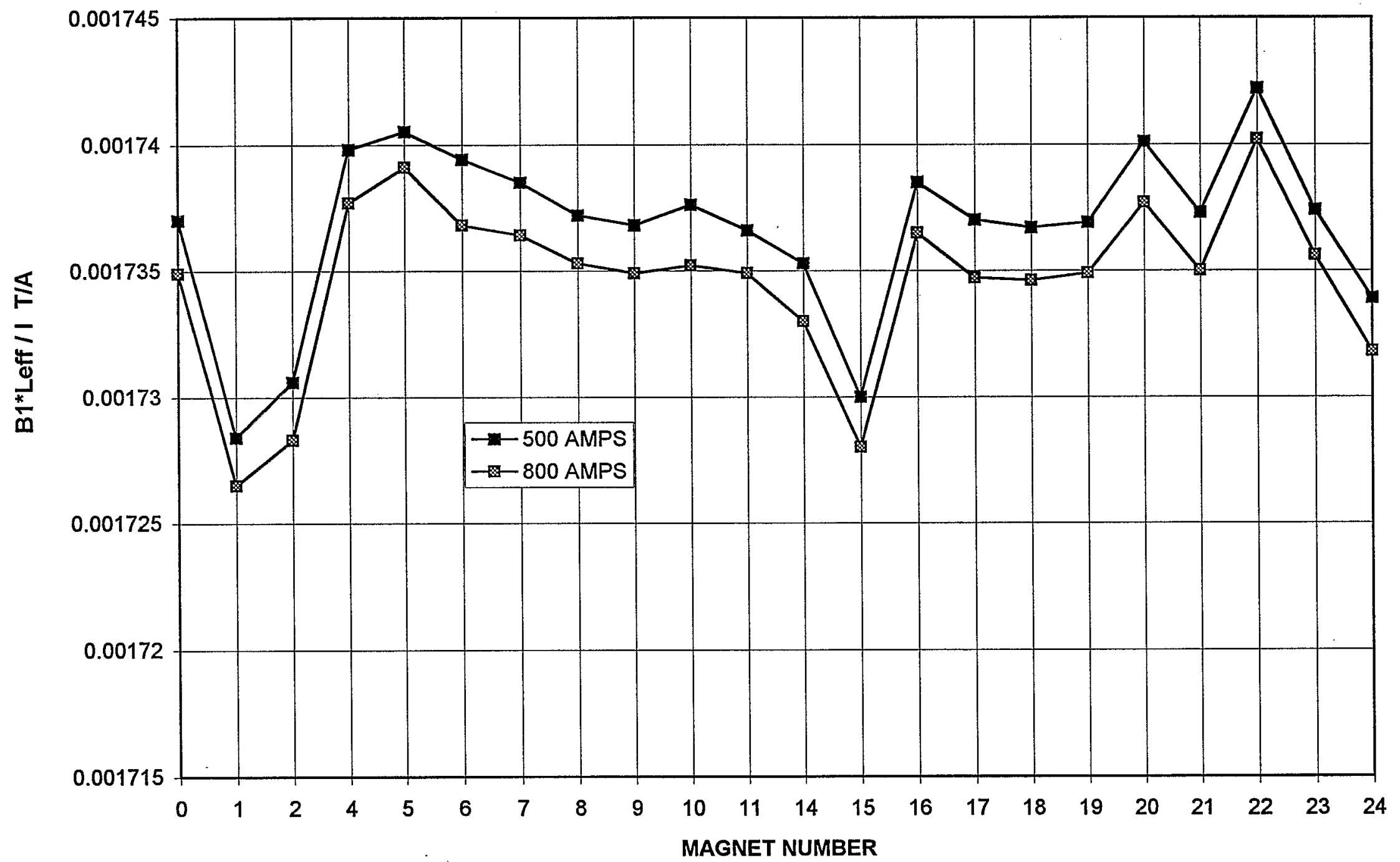



$\therefore$

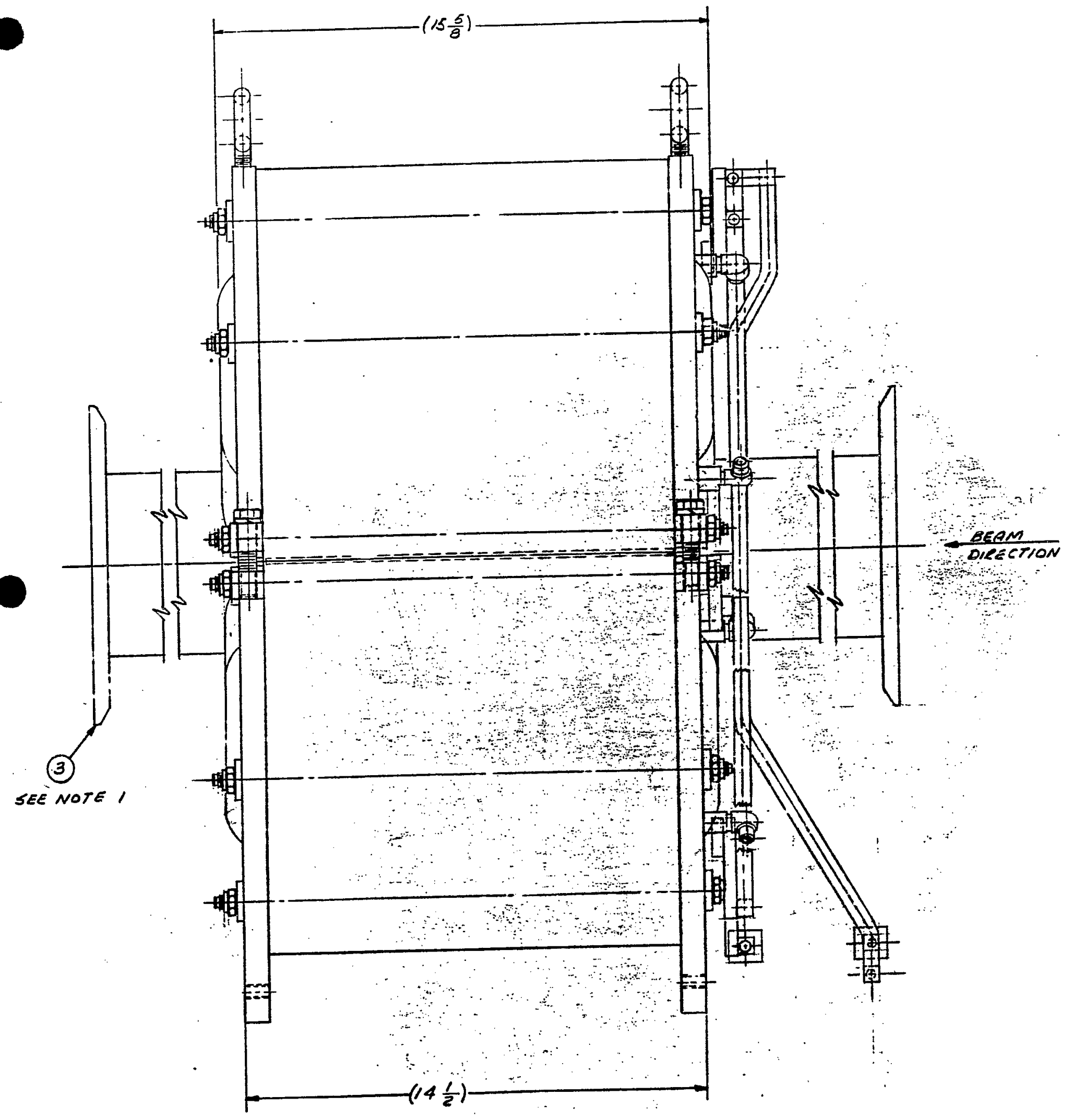


B. STANDARD MEASUREMENT REPORT

ANALYSIS Of FIELD SHAPE MEASUREMENTS

\begin{tabular}{|c|c|}
\hline MAGNET TYPE & AGS TUNE QUAD \\
\hline MAGNET NUMBER & QNU018 \\
\hline RUN NUMBER & QNU018.101 TX \\
\hline DATE of MEASUREMENT & 17-Mar-90 \\
\hline DATE of ANALYSIS & $11-F e b-92$ \\
\hline
\end{tabular}

SHORT SUMMARY Of MAGNET QUALITY

$======================================$

SUMMARY of QUADRUPOLE FIELD RESULTS

\begin{tabular}{|c|c|c|}
\hline B1* $L_{\text {eff }} / I @ 500$ Amps & $1.7367 \mathrm{E}-03$ & $T / A$ \\
\hline B1*Leff $/ 1 @ 800$ Amps & 1.7346E-03 & T/A \\
\hline
\end{tabular}

\begin{tabular}{|l|r|}
\hline SATURATION EFFECT & 1.00123 \\
\hline
\end{tabular}

[B1*Leff $/ 1 @ 500 \mathrm{Amps}] /\left[\mathrm{B} 1^{*} \mathrm{~L}_{\text {eff }} / \mathrm{l} @ 800 \mathrm{Amps}\right]$

SUMMARY of HARMONIC CONTENTS

\begin{tabular}{|l|r|r|c|}
\cline { 2 - 4 } \multicolumn{1}{c|}{} & \multicolumn{1}{c|}{ AVG } & \multicolumn{1}{c|}{ STD DEV } & UNITS \\
\hline B2/B1 & $-1.12 \mathrm{E}-02$ & $3.20 \mathrm{E}-04$ & $\mathrm{~m}^{-1}$ \\
\hline A2/B1 & $1.93 \mathrm{E}-03$ & $2.90 \mathrm{E}-04$ & $\mathrm{~m}^{-1}$ \\
\hline
\end{tabular}

\begin{tabular}{|l|l|l|l|}
\hline B3/B1 & $1.09 \mathrm{E}-01$ & $8.40 \mathrm{E}-03$ & $\mathrm{~m}^{-2}$ \\
\hline $\mathrm{A} 3 / \mathrm{B} 1$ & $7.00 \mathrm{E}-02$ & $1.30 \mathrm{E}-02$ & $\mathrm{~m}^{-2}$ \\
\hline
\end{tabular}

\begin{tabular}{|l|l|l|l|}
\hline $\mathrm{B} 4 / \mathrm{B} 1$ & $-2.78 \mathrm{E}-01$ & $9.30 \mathrm{E}-02$ & $\mathrm{~m}^{-3}$ \\
\hline $\mathrm{A} 4 / \mathrm{B} 1$ & $-1.87 \mathrm{E}-03$ & $1.20 \mathrm{E}-01$ & $\mathrm{~m}^{-3}$ \\
\hline
\end{tabular}

SUMMARY of ALIGNMENT PARAMETERS

\begin{tabular}{|l|r|r|c|}
\hline$x o$ & $2.71 E-04$ & $6.50 E-06$ & $m$ \\
\hline yo & $-1.33 E-04$ & $1.30 E-06$ & $m$ \\
\hline
\end{tabular}

\begin{tabular}{|l|l|l|l|}
\hline Theta & $-5.68 \mathrm{E}-04$ & $3.70 \mathrm{E}-05$ & radians \\
\hline
\end{tabular}

SUMMARY of RESIDUAL FIELDS

\begin{tabular}{|l|l|l|l|}
\hline$B 0^{*} L_{\text {eff }}$ & $6.75 \mathrm{E}-05$ & & $T^{*} \mathrm{~m}$ \\
\hline $\mathrm{A} 0^{*} \mathrm{~L}_{\text {eff }}$ & $1.70 \mathrm{E}-06$ & & $\mathrm{~T}^{*} \mathrm{~m}$ \\
\hline \multicolumn{3}{|l|}{} & \\
\hline $\mathrm{B} 1^{*} \mathrm{~L}_{\text {eff }}$ & $1.88 \mathrm{E}-03$ & & $\mathrm{~T}$ \\
\hline $\mathrm{A}{ }^{*} \mathrm{~L}_{\text {eff }}$ & $1.10 \mathrm{E}-04$ & & $\mathrm{~T}$ \\
\hline
\end{tabular}


BASIC MEASUREMENT RESULTS

\begin{tabular}{|c|c|c|c|c|c|c|}
\hline & 1 & $B 1^{*} L_{\text {eff }}$ & $B 0^{*} L_{\text {eff }}$ & B2* $L_{\text {eff }}$ & $B 3^{*} L_{\text {eff }}$ & $B 4^{*} L_{\text {eff }}$ \\
\hline & AMPS & $T$ & $T^{\star} m$ & $\mathrm{~T} / \mathrm{m}$ & $\mathrm{T} / \mathrm{m}^{2}$ & $\mathrm{~T} / \mathrm{m}^{3}$ \\
\hline 1 & 0.004 & 0.00188 & $6.750 \mathrm{E}-05$ & $-9.40 \mathrm{E}-05$ & $5.80 \mathrm{E}-04$ & $8.30 \mathrm{E}-02$ \\
\hline 2 & 24.529 & 0.04399 & $7.800 \mathrm{E}-05$ & -7.00 E-04 & $7.50 \mathrm{E}-03$ & $1.70 E-02$ \\
\hline 3 & 49.502 & 0.08702 & $8.900 \mathrm{E}-05$ & $-1.10 \mathrm{E}-03$ & $9.40 \mathrm{E}-03$ & $-8.10 \mathrm{E}-02$ \\
\hline 4 & 74.451 & 0.1301 & $9.910 \mathrm{E}-05$ & $-1.40 \mathrm{E}-03$ & $1.80 \mathrm{E}-02$ & $1.20 \mathrm{E}-01$ \\
\hline 5 & 99.373 & 0.17318 & $1.113 \mathrm{E}-04$ & $-2.10 \mathrm{E}-03$ & $1.50 \mathrm{E}-02$ & $-4.70 \mathrm{E}-02$ \\
\hline 6 & 199.141 & 0.34609 & $1.588 \mathrm{E}-04$ & $-3.70 \mathrm{E}-03$ & $3.80 \mathrm{E}-02$ & $-1.60 \mathrm{E}-01$ \\
\hline 7 & 298.774 & 0.51907 & $2.051 E-04$ & $-5.80 E-03$ & $6.00 \mathrm{E}-02$ & $-2.00 \mathrm{E}-01$ \\
\hline 8 & 398.529 & 0.69226 & $2.472 E-04$ & $-7.70 \mathrm{E}-03$ & $7.90 \mathrm{E}-02$ & $-1.20 \mathrm{E}-01$ \\
\hline 9 & 498.351 & 0.8655 & $2.905 \mathrm{E}-04$ & $-9.80 \mathrm{E}-03$ & $9.20 \mathrm{E}-02$ & $-1.80 \mathrm{E}-01$ \\
\hline 10 & 598.176 & 1.03842 & $3.413 \mathrm{E}-04$ & $-1.20 \mathrm{E}-02$ & $1.20 \mathrm{E}-01$ & $-3.00 \mathrm{E}-01$ \\
\hline 11 & 697.751 & 1.21097 & $3.855 \mathrm{E}-04$ & $-1.30 \mathrm{E}-02$ & $1.30 \mathrm{E}-01$ & $-2.50 \mathrm{E}-01$ \\
\hline 12 & 797.565 & 1.38345 & $4.284 \mathrm{E}-04$ & $-1.60 \mathrm{E}-02$ & $1.60 \mathrm{E}-01$ & $-3.30 \mathrm{E}-01$ \\
\hline
\end{tabular}

\begin{tabular}{|r|r|r|r|r|r|r|}
\multicolumn{1}{c|}{} & \multicolumn{1}{c|}{$\mathrm{A}$} & \multicolumn{1}{c|}{$\mathrm{A}{ }^{*} \mathrm{~L}_{\text {eff }}$} & \multicolumn{1}{c|}{$\mathrm{A} 0^{*} \mathrm{~L}_{\text {eff }}$} & \multicolumn{1}{c|}{$\mathrm{A} 2^{*} \mathrm{~L}_{\text {eff }}$} & $\mathrm{A}^{*} \mathrm{~L}_{\text {eff }}$ & \multicolumn{1}{c|}{$\mathrm{A}^{*} \mathrm{~L}_{\text {eff }}$} \\
\cline { 2 - 7 } & \multicolumn{1}{c|}{$\mathrm{AMPS}$} & $\mathrm{T}$ & $\mathrm{T} \mathrm{T}^{*} \mathrm{~m}$ & $\mathrm{~T} / \mathrm{m}$ & $\mathrm{T} / \mathrm{m}^{2}$ & $\mathrm{~T} / \mathrm{m}^{3}$ \\
\hline 1 & 0.004 & $1.10 \mathrm{E}-04$ & $1.70 \mathrm{E}-06$ & $-1.50 \mathrm{E}-05$ & $3.60 \mathrm{E}-03$ & $3.50 \mathrm{E}-02$ \\
\hline 2 & 24.529 & $9.00 \mathrm{E}-05$ & $-3.70 \mathrm{E}-06$ & $-1.30 \mathrm{E}-04$ & $3.60 \mathrm{E}-03$ & $-1.50 \mathrm{E}-01$ \\
\hline 3 & 49.502 & $6.00 \mathrm{E}-05$ & $-8.50 \mathrm{E}-06$ & $1.20 \mathrm{E}-04$ & $8.00 \mathrm{E}-03$ & $-5.50 \mathrm{E}-02$ \\
\hline 4 & 74.451 & $4.00 \mathrm{E}-05$ & $-1.54 \mathrm{E}-05$ & $5.80 \mathrm{E}-04$ & $1.40 \mathrm{E}-02$ & $-9.20 \mathrm{E}-04$ \\
\hline 5 & 99.373 & $1.00 \mathrm{E}-05$ & $-2.04 \mathrm{E}-05$ & $4.50 \mathrm{E}-04$ & $1.70 \mathrm{E}-02$ & $-1.90 \mathrm{E}-02$ \\
\hline 6 & 199.141 & $-7.00 \mathrm{E}-05$ & $-4.34 \mathrm{E}-05$ & $6.20 \mathrm{E}-04$ & $2.40 \mathrm{E}-02$ & $-2.40 \mathrm{E}-02$ \\
\hline 7 & 298.774 & $-1.90 \mathrm{E}-04$ & $-6.76 \mathrm{E}-05$ & $8.40 \mathrm{E}-04$ & $4.20 \mathrm{E}-02$ & $2.50 \mathrm{E}-02$ \\
\hline 8 & 398.529 & $-3.20 \mathrm{E}-04$ & $-9.04 \mathrm{E}-05$ & $1.40 \mathrm{E}-03$ & $4.80 \mathrm{E}-02$ & $1.90 \mathrm{E}-01$ \\
\hline 9 & 498.351 & $-3.80 \mathrm{E}-04$ & $-1.14 \mathrm{E}-04$ & $1.50 \mathrm{E}-03$ & $5.30 \mathrm{E}-02$ & $-5.60 \mathrm{E}-02$ \\
\hline 10 & 598.176 & $-5.00 \mathrm{E}-04$ & $-1.37 \mathrm{E}-04$ & $2.00 \mathrm{E}-03$ & $5.90 \mathrm{E}-02$ & $-1.00 \mathrm{E}-01$ \\
\hline 11 & 697.751 & $-6.10 \mathrm{E}-04$ & $-1.58 \mathrm{E}-04$ & $2.50 \mathrm{E}-03$ & $7.80 \mathrm{E}-02$ & $1.20 \mathrm{E}-01$ \\
\hline 12 & 797.565 & $-7.60 \mathrm{E}-04$ & $-1.84 \mathrm{E}-04$ & $2.30 \mathrm{E}-03$ & $8.10 \mathrm{E}-02$ & $-1.20 \mathrm{E}-01$ \\
\hline
\end{tabular}


GRADIENT and POSITION ANALYSIS

Residual Field Subtracted

\begin{tabular}{|c|c|c|c|c|c|c|}
\hline \multicolumn{7}{|c|}{ 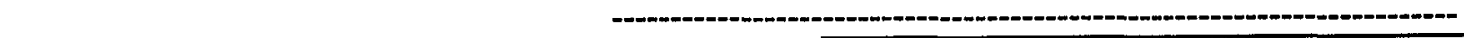 } \\
\hline & & & & Theta & $x 0$ & yo \\
\hline & 1 & $\overline{B 1 * L_{e f f} / I}$ & $B 1^{*} L_{\text {eff }} / I$ & $\mathrm{~A} 1 / \mathrm{B} 1$ & B0/B1 & A0/B1 \\
\hline & AMPS & T/A & $T / A$ & radians & $m$ & $m$ \\
\hline 1 & 0.004 & & & & & \\
\hline 2 & 24.529 & $1.7933 \mathrm{E}-03$ & $1.7155 \mathrm{E}-03$ & $-3.24 \mathrm{E}-04$ & $3.58 \mathrm{E}-04$ & $-1.37 E-04$ \\
\hline 3 & 49.502 & $1.7579 \mathrm{E}-03$ & $1.7194 \mathrm{E}-03$ & $-5.32 E-04$ & $3.06 \mathrm{E}-04$ & $-1.24 \mathrm{E}-04$ \\
\hline 4 & 74.451 & $1.7474 \mathrm{E}-03$ & $1.7218 \mathrm{E}-03$ & $-5.03 E-04$ & $2.82 \mathrm{E}-04$ & $-1.36 \mathrm{E}-04$ \\
\hline 5 & 99.373 & $1.7427 \mathrm{E}-03$ & $1.7235 \mathrm{E}-03$ & $-5.26 E-04$ & $2.82 E-04$ & $-1.31 E-04$ \\
\hline 6 & 199.141 & $1.7379 E-03$ & $1.7283 \mathrm{E}-03$ & $-5.03 E-04$ & $2.78 \mathrm{E}-04$ & $-1.32 E-04$ \\
\hline 7 & 298.774 & $1.7373 \mathrm{E}-03$ & $1.7309 \mathrm{E}-03$ & $-5.62 E-04$ & $2.75 \mathrm{E}-04$ & $-1.35 E-04$ \\
\hline 8 & 398.529 & $1.7370 \mathrm{E}-03$ & $1.7323 \mathrm{E}-03$ & $-6.04 \mathrm{E}-04$ & 2.67E-04 & $-1.34 \mathrm{E}-04$ \\
\hline 9 & 498.351 & $1.7367 \mathrm{E}-03$ & $1.7329 \mathrm{E}-03$ & $-5.58 E-04$ & $2.64 \mathrm{E}-04$ & $-1.34 E-04$ \\
\hline 10 & 598.176 & 1.7360E-03 & $1.7328 \mathrm{E}-03$ & $-5.85 E-04$ & $2.69 \mathrm{E}-04$ & $-1.34 \mathrm{E}-04$ \\
\hline 11 & 697.751 & $1.7355 \mathrm{E}-03$ & $1.7328 \mathrm{E}-03$ & $-5.87 E-04$ & $2.67 \mathrm{E}-04$ & $-1.32 E-04$ \\
\hline 12 & 797.565 & 1.7346E-03 & $1.7322 \mathrm{E}-03$ & $-6.24 E-04$ & $2.65 \mathrm{E}-04$ & $-1.34 E-04$ \\
\hline & AVERAG & $(100$ to $800 \mathrm{~A}$ & $\mathrm{nps)}=$ & $-5.68 \mathrm{E}-04$ & $2.71 \mathrm{E}-04$ & $-1.33 \mathrm{E}-04$ \\
\hline & STANDAI & D DEVIATIOI & $=$ & $3.70 \mathrm{E}-05$ & $6.50 \mathrm{E}-06$ & $1.30 \mathrm{E}-06$ \\
\hline
\end{tabular}


HARMONIC ANALYSIS

$======= \pm======$

\begin{tabular}{|r|r|r|r|r|r|r|}
\cline { 2 - 7 } \multicolumn{1}{c|}{} & \multicolumn{1}{c|}{$\mathrm{I}$} & $\mathrm{B} 1{ }^{*} \mathrm{~L}_{\text {eff }} / \mathrm{l}$ & $\mathrm{B} 0 / \mathrm{B} 1$ & $\mathrm{~B} 2 / \mathrm{B} 1$ & $\mathrm{~B} 3 / \mathrm{B} 1$ & \multicolumn{1}{c|}{$\mathrm{B} 4 / \mathrm{B} 1$} \\
\cline { 2 - 7 } \multicolumn{1}{c|}{} & \multicolumn{1}{c|}{$\mathrm{AMPS}$} & $\mathrm{T} / \mathrm{A}$ & $\mathrm{m}$ & $\mathrm{m}^{-1}$ & $\mathrm{~m}^{-2}$ & $\mathrm{~m}^{-3}$ \\
\hline 1 & 0.004 & $4.6900 \mathrm{E}-01$ & $3.60 \mathrm{E}-02$ & $-5.00 \mathrm{E}-02$ & $3.12 \mathrm{E}-01$ & $4.40 \mathrm{E}+01$ \\
\hline 2 & 24.529 & $1.7933 \mathrm{E}-03$ & $1.77 \mathrm{E}-03$ & $-1.59 \mathrm{E}-02$ & $1.71 \mathrm{E}-01$ & $3.80 \mathrm{E}-01$ \\
\hline 3 & 49.502 & $1.7579 \mathrm{E}-03$ & $1.02 \mathrm{E}-03$ & $-1.32 \mathrm{E}-02$ & $1.08 \mathrm{E}-01$ & $-9.30 \mathrm{E}-01$ \\
\hline 4 & 74.451 & $1.7474 \mathrm{E}-03$ & $7.60 \mathrm{E}-04$ & $-1.10 \mathrm{E}-02$ & $1.35 \mathrm{E}-01$ & $9.30 \mathrm{E}-01$ \\
\hline 5 & 99.373 & $1.7427 \mathrm{E}-03$ & $6.40 \mathrm{E}-04$ & $-1.19 \mathrm{E}-02$ & $8.94 \mathrm{E}-02$ & $-2.70 \mathrm{E}-01$ \\
\hline 6 & 199.141 & $1.7379 \mathrm{E}-03$ & $4.60 \mathrm{E}-04$ & $-1.07 \mathrm{E}-02$ & $1.08 \mathrm{E}-01$ & $-4.70 \mathrm{E}-01$ \\
\hline 7 & 298.774 & $1.7373 \mathrm{E}-03$ & $4.00 \mathrm{E}-04$ & $-1.13 \mathrm{E}-02$ & $1.15 \mathrm{E}-01$ & $-3.80 \mathrm{E}-01$ \\
\hline 8 & 398.529 & $1.7370 \mathrm{E}-03$ & $3.60 \mathrm{E}-04$ & $-1.11 \mathrm{E}-02$ & $1.14 \mathrm{E}-01$ & $-1.70 \mathrm{E}-01$ \\
\hline 9 & 498.351 & $1.7367 \mathrm{E}-03$ & $3.40 \mathrm{E}-04$ & $-1.13 \mathrm{E}-02$ & $1.06 \mathrm{E}-01$ & $-2.00 \mathrm{E}-01$ \\
\hline 10 & 598.176 & $1.7360 \mathrm{E}-03$ & $3.30 \mathrm{E}-04$ & $-1.11 \mathrm{E}-02$ & $1.18 \mathrm{E}-01$ & $-2.90 \mathrm{E}-01$ \\
\hline 11 & 697.751 & $1.7355 \mathrm{E}-03$ & $3.20 \mathrm{E}-04$ & $-1.11 \mathrm{E}-02$ & $1.08 \mathrm{E}-01$ & $-2.10 \mathrm{E}-01$ \\
\hline 12 & 797.565 & $1.7346 \mathrm{E}-03$ & $3.10 \mathrm{E}-04$ & $-1.14 \mathrm{E}-02$ & $1.13 \mathrm{E}-01$ & $-2.40 \mathrm{E}-01$ \\
\hline
\end{tabular}

\begin{tabular}{|l|r|r|r|}
\hline AVERAGE $(100$ to $800 \mathrm{Amps})=$ & $-1.12 \mathrm{E}-02$ & $1.09 \mathrm{E}-01$ & $-2.78 \mathrm{E}-01$ \\
\hline STANDARD DEVIATION $=$ & $3.20 \mathrm{E}-04$ & $8.40 \mathrm{E}-03$ & $9.30 \mathrm{E}-02$ \\
\hline
\end{tabular}

\begin{tabular}{|r|r|r|r|r|r|r|}
\cline { 2 - 7 } \multicolumn{1}{c|}{} & \multicolumn{1}{c|}{$\mathrm{I}$} & $\mathrm{A} 1 / \mathrm{B} 1$ & $\mathrm{~A} / \mathrm{A} / \mathrm{B} 1$ & $\mathrm{~A} 2 / \mathrm{B} 1$ & $\mathrm{~A} 3 / \mathrm{B} 1$ & \multicolumn{1}{c|}{$\mathrm{A} 4 / \mathrm{B} 1$} \\
\cline { 2 - 7 } \multicolumn{1}{c|}{} & AMPS & radians & $\mathrm{m}$ & $\mathrm{m}^{-1}$ & $\mathrm{~m}^{-2}$ & $\mathrm{~m}^{-3}$ \\
\hline 1 & 0.004 & $6.10 \mathrm{E}-02$ & 0.00088 & $-8.25 \mathrm{E}-03$ & $1.89 \mathrm{E}+00$ & $1.90 \mathrm{E}+01$ \\
\hline 2 & 24.529 & $2.00 \mathrm{E}-03$ & -0.00009 & $-2.89 \mathrm{E}-03$ & $8.20 \mathrm{E}-02$ & $-3.40 \mathrm{E}+00$ \\
\hline 3 & 49.502 & $6.50 \mathrm{E}-04$ & -0.0001 & $1.41 \mathrm{E}-03$ & $9.16 \mathrm{E}-02$ & $-6.30 \mathrm{E}-01$ \\
\hline 4 & 74.451 & $2.90 \mathrm{E}-04$ & -0.00012 & $4.48 \mathrm{E}-03$ & $1.10 \mathrm{E}-01$ & $-7.00 \mathrm{E}-03$ \\
\hline 5 & 99.373 & $6.70 \mathrm{E}-05$ & -0.00012 & $2.58 \mathrm{E}-03$ & $9.88 \mathrm{E}-02$ & $-1.10 \mathrm{E}-01$ \\
\hline 6 & 199.141 & $-2.10 \mathrm{E}-04$ & -0.00013 & $1.79 \mathrm{E}-03$ & $7.01 \mathrm{E}-02$ & $-6.80 \mathrm{E}-02$ \\
\hline 7 & 298.774 & $-3.60 \mathrm{E}-04$ & -0.00013 & $1.62 \mathrm{E}-03$ & $8.03 \mathrm{E}-02$ & $4.90 \mathrm{E}-02$ \\
\hline 8 & 398.529 & $-4.60 \mathrm{E}-04$ & -0.00013 & $2.05 \mathrm{E}-03$ & $6.86 \mathrm{E}-02$ & $2.70 \mathrm{E}-01$ \\
\hline 9 & 498.351 & $-4.40 \mathrm{E}-04$ & -0.00013 & $1.79 \mathrm{E}-03$ & $6.17 \mathrm{E}-02$ & $-6.40 \mathrm{E}-02$ \\
\hline 10 & 598.176 & $-4.90 \mathrm{E}-04$ & -0.00013 & $1.92 \mathrm{E}-03$ & $5.72 \mathrm{E}-02$ & $-1.00 \mathrm{E}-01$ \\
\hline 11 & 697.751 & $-5.00 \mathrm{E}-04$ & -0.00013 & $2.05 \mathrm{E}-03$ & $6.44 \mathrm{E}-02$ & $1.00 \mathrm{E}-01$ \\
\hline 12 & 797.565 & $-5.50 \mathrm{E}-04$ & -0.00013 & $1.68 \mathrm{E}-03$ & $5.87 \mathrm{E}-02$ & $-8.90 \mathrm{E}-02$ \\
\hline
\end{tabular}

\begin{tabular}{|l|r|r|r|}
\hline AVERAGE $(100$ to 800 Amps $)=$ & $1.93 \mathrm{E}-03$ & $7.00 \mathrm{E}-02$ & $-1.87 \mathrm{E}-03$ \\
\hline STANDARD DEVIATION $=$ & $2.90 \mathrm{E}-04$ & $1.30 \mathrm{E}-02$ & $1.20 \mathrm{E}-01$ \\
\hline
\end{tabular}




\section{PARAMETER SHEET FOR THE AGS TUNE QUAD}

Issue Date: October 25, 1996

PROTOTYPE NAME

MAGNET CLASS

NUMBER OF MAGNETS
QNU (AGS TUNE QUAD \& SKEW QUAD)

QUADRUPOLE (Uses booster quad laminations)

30

\section{MECHANICAL}

CORE

LAMINATION LENGTH TOLERANCE SPECIFIED TOLERANCE MEASURED

STRUCTURAL LENGTH

COIL LENGTH

OVERALL LENGTH

APERTURE SHAPE

RADIUS AT POLE TIP TOLERANCE SPECIFIED TOLERANCE MEASURED

POLE WIDTH

CORE HEIGHT

CORE WIDTH

\section{LAMINATIONS}

MATERIAL

COATING

COATING THICKNESS

OVERALL THICKNESS

APPROX LAMS PER BLOCK

QUADRANT BLOCK WEIGHT TOLERANCE SPECIFIED TOLERANCE MEASURED

\section{VACUUM PIPE}

HEIGHT - OUTSIDE

WIDTH - OUTSIDE

WALL THICKNESS

TOLERANCE SPECIFIED

TOLERANCE MEASURED

HALF HEIGHT - INSIDE

HALF WIDTH - INSIDE

MATERIAL

RESISTIVITY

TOLERANCE SPECIFIED

TOLERANCE MEASURED

INCHES
\begin{tabular}{|r|r|}
\hline 13 & MILLIMETERS \\
\hline 0.003 & 330.2 \\
\hline$\# \mathrm{~N} / \mathrm{A}$ & 0.076 \\
\hline 14.5 & $\# \mathrm{~N} / \mathrm{A}$ \\
\hline 15.625 & 368.3 \\
\hline 17.5 & 396.9 \\
\hline \multicolumn{2}{|c|}{ ROUND } \\
\hline 3.25 & 444.5 \\
\hline 0.003 & 0.076 \\
\hline 0.002 & 0.051 \\
\hline 5.142 & 130.6 \\
\hline 23.15 & 588.0 \\
\hline 23.15 & 588.0 \\
\hline
\end{tabular}

REF

a

a

a

a

a

a

a

a

a

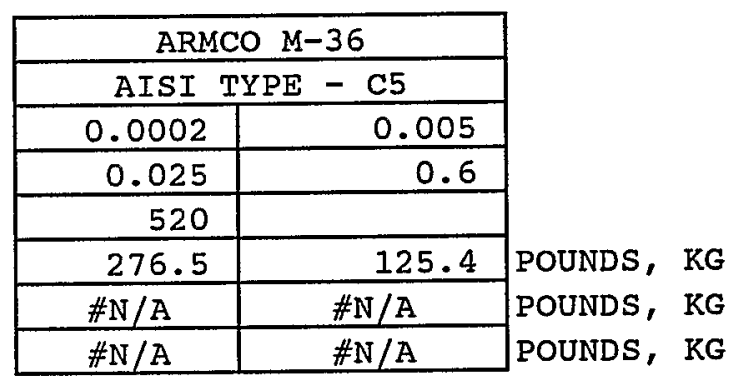

a

a

a

a

a

a

b

b

b

b

$\mathrm{b}$

b

$\# \mathrm{~N} / \mathrm{A}$

$\# N / A$
MICRO-OHM CM b MICRO-OHM CM b 


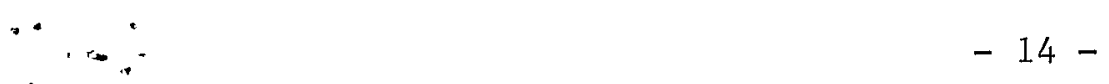

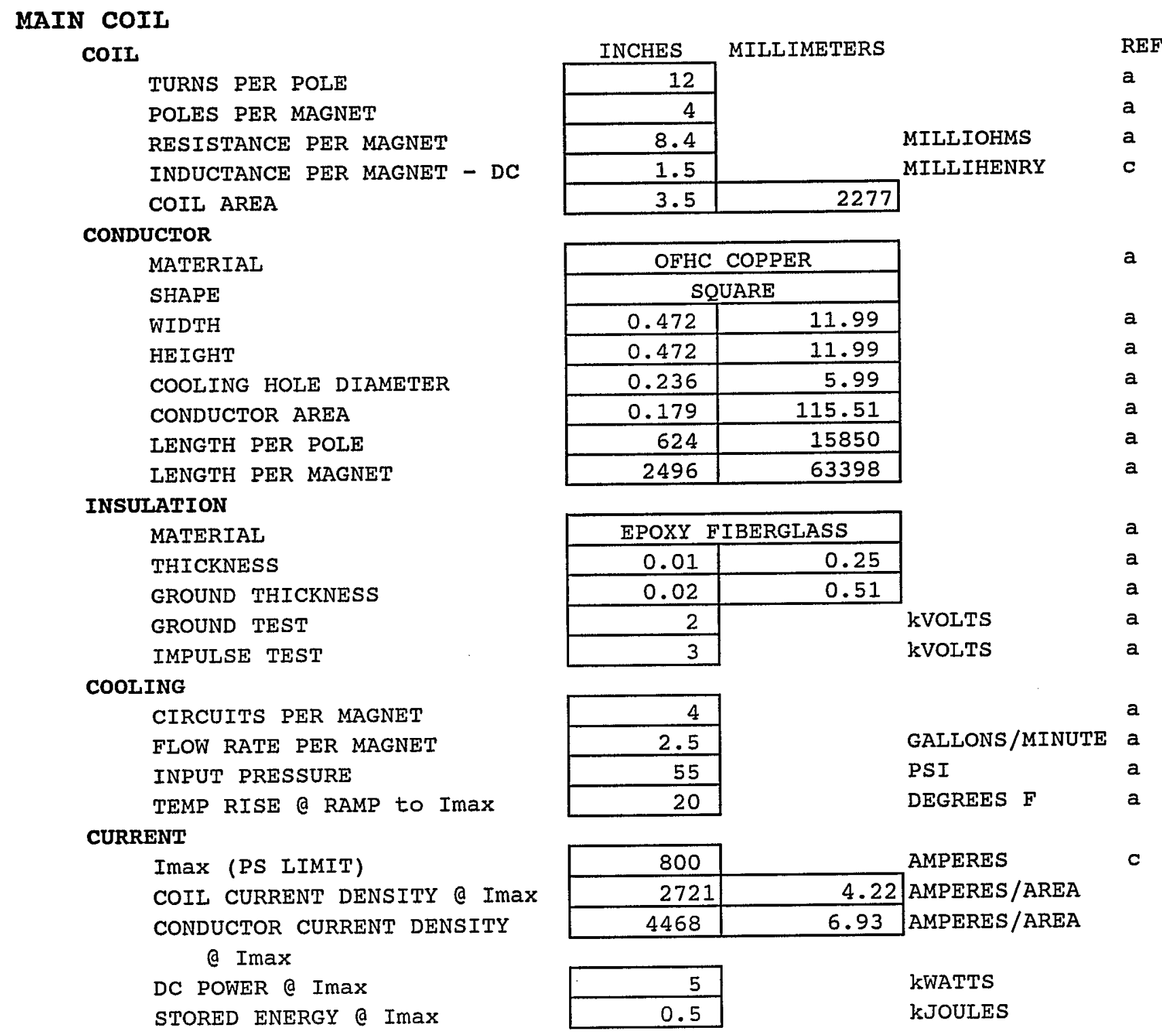


$10 .+15-$

TUNE TRIM COIL

COIL

TURNS PER POLE

POLES PER MAGNET

RESISTANCE PER MAGNET

INDUCTANCE PER MAGNET - DC

CONDUCTOR

MATERIAL

SHAPE

DIAMETER

AREA

LENGTH PER POLE

LENGTH PER MAGNET

INSULATION

MATERIAL

THICKNESS

GROUND TEST

IMPULSE TEST

CURRENT

Imax (PS LIMIT)

CURRENT DENSITY @ Imax

DC POWER @ Imax

STORED ENERGY @ Imax

\begin{tabular}{|c|c|}
\hline INCHES & \multirow{2}{*}{ MILLIMETERS } \\
\hline 12 & \\
\hline 4 & \\
\hline 208 & \\
\hline 1.5 & \\
\hline$\# 10$ COPP & WIRE - ETP \\
\hline \multicolumn{2}{|c|}{ ROUND } \\
\hline 0.101 & 2.57 \\
\hline 0.008155 & 5.3 \\
\hline 624 & 15850 \\
\hline 2496 & 63398 \\
\hline
\end{tabular}

REF

a

MILLIOHMS

MILLIHENRY C

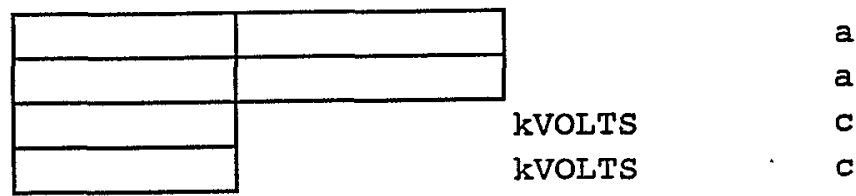

\begin{tabular}{|c|c|c|}
\hline 10 & & AMPERES \\
\hline 1226 & 1.9 & AMPERES/AREA \\
\hline 20.80 & & WATTS \\
\hline 0.1 & & JOULES \\
\hline
\end{tabular}




\section{MAGNETIC PROPERTIES OF THE MAIN COIL}

RANDOM ERRORS

$B 1 *$ Leff/I rms

0.000003

REF

CAL MEASUREMENTS

TYPICAL MEASUREMENTS
B1*Leff @ I $=0$

$\mathrm{T} / \mathrm{A}$

e

$B I *$ Leff / I

Q200 AMPS

Q400 AMPS

Q600 AMPS

@800 AMPS

\begin{tabular}{|l|}
\hline 0.001880 \\
\hline 0.001738 \\
\hline 0.001737 \\
\hline 0.001736 \\
\hline 0.001735 \\
\hline
\end{tabular}

CALCULATIONS

B1/I
Leff
@200 AMPS
$@ 400$ AMPS
$@ 600$ AMPS
@800 AMPS
POLE TIP FIELD
@200 AMPS
@400 AMPS
@600 AMPS
@800 AMPS

0.004426

$[\mathrm{T} / \mathrm{m}] / \mathrm{A}$

e

meters e

meters e

meters $e$

0.392

0.392

meters e

$7.31 \mathrm{E}-02$

$1.46 \mathrm{E}-01$

$2.19 \mathrm{E}-01$

$2.92 \mathrm{E}-01$

$\begin{array}{ll}T & e \\ T & e \\ T & e \\ T & e\end{array}$

MAGNETIC PROPERTIES OF THE TUNE TRIM COIL CALCULATIONS
$B 1 / I$
$B I *$ Leff $/ I$
\begin{tabular}{|l|}
\hline 0.004426 \\
\hline 0.001738 \\
\hline
\end{tabular}

$\underset{T / A}{T / \mathrm{m}] / \mathrm{A}}$

e

e

e

e

e

e

e

\section{REFERENCES}

a. E. RODGER, Private communication

b. H. C. HSUEH, Private communication

c. A. SOUKAS, Private communication

e. E. BLESER 